\title{
Focus on treatment of lung carcinoid tumor
}

This article was published in the following Dove Press journal:

OncoTargets and Therapy

24 October 2013

Number of times this article has been viewed

\section{Elise Noel-Savina' \\ Renaud Descourt ${ }^{2}$ \\ 'Pulmonary Service, ${ }^{2}$ Thoracic Oncology Service, Hospital de la Cavale Blanche, $\mathrm{CHU}$ - Brest, Brest, France}

\begin{abstract}
Bronchial typical carcinoid tumors are neuroendocrine bronchopulmonary tumors with a low-grade malignancy, and an atypical carcinoid is an intermediate form of these tumors. There is a lack of knowledge on the optimal treatment for these tumors. The surgical treatment of choice consists of a lobectomy supplemented by dissection. The benefit of chemotherapy and radiotherapy is unclear. Targeted therapy could be used in this condition, but there is a lack of research recommending it.
\end{abstract}

Keywords: carcinoid tumor, neuroendocrine tumor, bronchopulmonary tumor, treatment

\section{Introduction}

Carcinoid tumors account for approximately $2 \%$ of all bronchopulmonary tumors. Bronchial typical carcinoid tumors are neuroendocrine bronchopulmonary tumors with a low-grade malignancy. An atypical carcinoid is an intermediate form of these tumors. Small and large cell neuroendocrine lung carcinomas represent a high-grade malignancy. There is a lack of knowledge on the optimal treatment of these tumors.

These entities are distinguished by their clinical, radiological and histological presentation, and - above all - their prognosis. Typical carcinoid tumors, which represent $90 \%$ of carcinoid lung neoplasms, occur mostly in young patients. Typical carcinoid tumors represent $1 \%-2 \%$ of lung tumors. In addition, $5 \%-15 \%$ have lymph node metastasis at presentation and 3\% have distant metastasis. Atypical carcinoid tumors represent $0.1 \%-0.2 \%$ of lung tumors, $40 \%-50 \%$ with lymph nodes metastasis at presentation, and $20 \%$ with distant metastasis. ${ }^{1}$

Depending on the series, cases are asymptomatic and discovered incidentally. In symptomatic patients, the time between the first signs and diagnosis can be long. The symptoms are chronic cough, chest pain, dyspnea, hemoptysis, fever, unilateral wheezing, or recurrent infections. The symptomatology is more meaningful when the tumor is proximal. In about $1 \%-5 \%$ of cases, carcinoid syndrome can be observed and is secondary to the systemic release of vasoactive substances, particularly serotonin. ${ }^{2}$

Radiologically, there are no specific signs. When the tumor is central, it is in the form of a hilar or perihilar mass, alone or in charge of a ventilatory defect downstream. In $16 \%-40 \%$ of cases, the tumor is peripheral and corresponds to a round or oval homogeneous opacity. Nearly $60 \%$ of carcinoid tumors are located in the right lung, the middle lobe being the favorite site. Computed tomography is an important test to clarify the radiological aspect observed on the chest radiography. In almost 30\% of cases, localized or diffuse calcifications may be visible. Bronchoscopy highlights budding 
well-vascularized lesions in three-fourths of the cases. Bronchial biopsies exposed to a significant risk of bleeding and caution must be set against tumors that are endoscopically well-rounded and raspberry in color.

In addition, the small size of bronchial biopsies often poses diagnostic problems. The main histologic features for distinguishing among lung neuroendocrine tumors are the morphology, the mitotic account, and the presence or absence of necrosis. Typical and atypical carcinoid tumors have an identical morphology. The mitotic account with more than one mitosis to ten high-power fields for typical carcinoid (two to ten mitoses for an atypical carcinoid) and the absence of necrosis distinguish the typical and the atypical carcinoid, according to the classification of Travis, ${ }^{3}$ behind the World Health Organization classification of 1999. The neuroendocrine tumors would distinguish them also by the variable expression of genetic markers linked to the p53 locus of chromosome 17p13. Some immunochemical markers characterize the neuroendocrine tumor: chromogranin, synaptophysin, and neural cell adhesion molecule/CD56. The thyroid transcription factor 1 is negative.

The treatment of choice of these carcinoid tumors is surgical and usually consists of a lobectomy, supplemented by lymph node dissection. In inoperable cases, patients may be offered endoscopic resection. But problems arise in this case with the absence of lymph node staging. The benefit of chemotherapy is not clear, but this question arises only in the case of metastatic disease.

Most carcinoid tumors have a benign course. It is a slowgrowing tumor. Some more aggressive tumors may develop metastasis. Depending on the series, the typical forms metastasize in $2 \%-11 \%$ of cases and very rarely more. ${ }^{4}$ The survival of patients with carcinoid tumors depends mainly on the histological type and the presence of metastasis. ${ }^{5} \mathrm{We}$ focused on the systemic and the local treatment of lung carcinoid tumor, not on a local therapy of metastasis.

\section{Treatment}

\section{Surgery}

Surgery is the mainstay of the treatment, based on the general principle of complete resection with preservation of as much normal lung tissue as possible.

A study conducted by Ducrocq ${ }^{6}$ examined factors determining long-term survival after resection of typical carcinoid tumors. It failed to demonstrate any prognostic significance for sex, tumor size (T1 versus T2), tumor location (central versus peripheral), and type of resection. The study confirms a good prognosis after complete resection of typical carcinoid tumors, including those with lymph node metastasis. Parenchyma-saving resections should be preferred for a typical carcinoid tumor. ${ }^{7}$ Patients who have an atypical carcinoid tumor with a consequent higher rate of nodal involvement should receive a radical surgery. ${ }^{6,8}$ Published in 2010, a retrospective study of 126 patients treated surgically for a carcinoid tumor showed that the survival at 10 years was $79.8 \%$. The main prognostic factors are histology (with a significantly higher survival for typical carcinoid tumors), lymph node, and metastasis. ${ }^{9}$ In 2007, in another study of 661 patients treated surgically for a carcinoid tumor, the overall five-year survival was $97 \%$ for typical carcinoid tumors (100\% for those with nodal involvement, but no significant difference) and $78 \%$ for atypical carcinoid tumors $(60 \%$ with positive lymph nodes, with a statistically significant difference). The histology and lymph node involvement were the main prognostic factors for these patients. Lymph node dissection during surgery is important. ${ }^{10}$ Endobronchial removal should be reserved for very selected typical carcinoid tumor with endobronchial structures without an extension through the cartilaginous area. ${ }^{11}$

\section{Chemotherapy and radiotherapy}

Chemotherapy should be considered in two cases: adjuvant or neoadjuvant therapy with surgery and for advanced disease.

Adjuvant chemotherapy has not been well-studied in this indication. Those patients received adjuvant chemotherapy with lymph node involvement and treated with surgical resection alone were more likely to develop recurrent disease and a significantly worse outcome. The data just concerned large cell neuroendocrine carcinoma and small cell carcinoma; there was a lack of knowledge for adjuvant chemotherapy. The use of the chemotherapy regimens usually administered in small cell lung cancer therapy, including platinum plus etoposide, should be an option like large cell neuroendocrine carcinoma. ${ }^{12}$ Radiotherapy could control the local disease. In the presence of the advanced pulmonary carcinoids, several chemotherapeutic agents have been investigated, but they were generally associated with discouraging results. There is a lack of randomized trials, especially placebo-controlled studies. In a study by Sun et al, ${ }^{13}$ patients with advanced carcinoid tumors were randomized to either doxorubicin with fluorouracil or fluorouracil with streptozocin. Responses to this treatment were modest. Fluorouracil with streptozocin improved survival (but not the response rates and the progression-free survival [PFS]) compared with the doxorubicin-based regimen, suggesting 
that it should be considered an active regimen of therapy. Other studies evaluated somatostatin analogs (octreotide or lanreotide) and 5-fluorouracil, dacarbazine, epi-adriamycin, oxaliplatin plus capecitabine, and temozolomide.

The RAD001 In Advanced Neuroendocrine Tumors-2 (RADIANT-2) study evaluated ${ }^{14}$ everolimus, an oral inhibitor of the mammalian target of rapamycin, plus octreotide long-acting repeatable (LAR) in patients with low-grade or intermediate-grade neuroendocrine advanced tumors (carcinoids). Everolimus plus octreotide LAR, compared with a placebo plus octreotide LAR, improved PFS in patients with carcinoid syndrome.

Some peptide and amine markers (chromogranin A, neuron-specific enolase, serotonin, synaptophysin, and adrenocorticotropic hormone) should be used to make the differential diagnosis. Somatostatin receptors (SSTRs) are involved in the different intracellular signaling pathways of cell proliferation, differentiation, and angiogenesis. The presence of SSTRs should be demonstrated to justify the patient's selection for somatostatin analog therapy.

A high heterogeneity was reported for the SSTRs' distribution, with a significant progressive decrease from low- to high-grade forms. The somatostatin analog therapy mimicked the actions of somatostatin. Octreotide has been used for its antisecretory effects and has proven to be an effective agent for symptoms of carcinoid syndrome. Lanreotide is a synthetic analog of somatostatin. Several results supported the recommendation for the use of somatostatin analogs in treating carcinoid syndrome, ${ }^{15,16}$ but any other use, including the management of asymptomatic metastatic carcinoids, can still be considered controversial. A relatively new therapy is peptide receptor radionuclide therapy with radiolabeled somatostatin analogs (90Y-DOTATOC and $177 \mathrm{Lu}-$ DOTATATE. 90Y-DOTATOC). These new treatments were not actually evaluated in only bronchopulmonary carcinoid tumors by randomized study, but the data in all carcinoid tumors seemed to be interesting in some Phase II clinical studies, ${ }^{17,18}$ with few adverse events and very interesting outcomes in patients with carcinoid tumors with few or no alternative treatments.

\section{Targeted therapy}

Targeted therapies were not well-evaluated in neuroendocrine tumors with pulmonary origin. In carcinoid tumors, overexpression of the vascular endothelial growth factor (VEGF), together with the VEGF receptor (VEGFR) subtypes, have been observed. This suggested that an autocrine activation of the VEGF pathway may promote tumor growth. Sunitinib malate is a small molecule kinase inhibitor with activity against a number of tyrosine kinase receptors (VEGFR-1, VEGFR-2, VEGFR-3, platelet-derived growth factor receptors, stem cell factor receptors, glial cell line-derived neurotrophic factor receptors, and fms-like tyrosine kinase-3). The efficacy of sunitinib has been evaluated in a two-cohort Phase II clinical study of advanced carcinoid and pancreatic neuroendocrine tumor patients. In spite of the antitumor activity in pancreatic neuroendocrine tumors, its activity against carcinoid tumors could not be definitively determined in the study. ${ }^{19}$

Interferon-alpha has been described to have antiangiogenic properties and has also been studied in carcinoids. Adding interferon-alpha to octreotide may retard tumor growth in patients with midgut carcinoid tumors. ${ }^{20}$

The combination of bevacizumab (BEV) and pegylated (PEG) interferon alpha-2b was evaluated among patients with metastatic or unresectable carcinoid tumors. Patients on stable doses of octreotide were randomly assigned to 18 weeks of treatment with BEV or PEG interferon alpha- $2 b$. $\mathrm{BEV}$ therapy resulted in objective responses, with reduction of tumor blood flow, and longer PFS in patients with carcinoid tumors than PEG interferon treatment. The results reported with BEV can be considered very interesting, but they need to be confirmed in larger studies. ${ }^{21}$

Rickman et $\mathrm{a}^{22}$ investigated the expression of the ErbB family of receptor tyrosine kinases in pulmonary - typical and atypical - carcinoid tumors and evaluated the role of epidermal growth factor receptor (EGFR) signaling in tumor proliferation. $45.8 \%$ of typical carcinoid and $28.6 \%$ of atypical carcinoid tumors express EGFR, $100 \%$ of the tumors lack expression of ErbB2, and 100\% have moderate-to-intense staining for ErbB3 and ErbB4. No EGFR mutations were found, and they sequenced the KRAS gene from the pulmonary carcinoid tumor DNA and found that $100 \%$ were wild-type. Moreover, using a lung carcinoid cell line that expresses EGFR, they found that erlotinib reduced proliferation by inhibiting the EGFR signal transduction. The results suggested a clinical potential for the use of EGFR inhibitors in the treatment of patients with pulmonary carcinoid tumors, particularly for those patients who were not amenable to surgical resection..$^{22}$

\section{Metastasis}

For extrahepatic metastasis, the treatment is systemic therapy. For hepatic metastasis, local treatment could be used - metastasis surgery, hepatic artery embolization, or chemoembolization. ${ }^{23}$ 


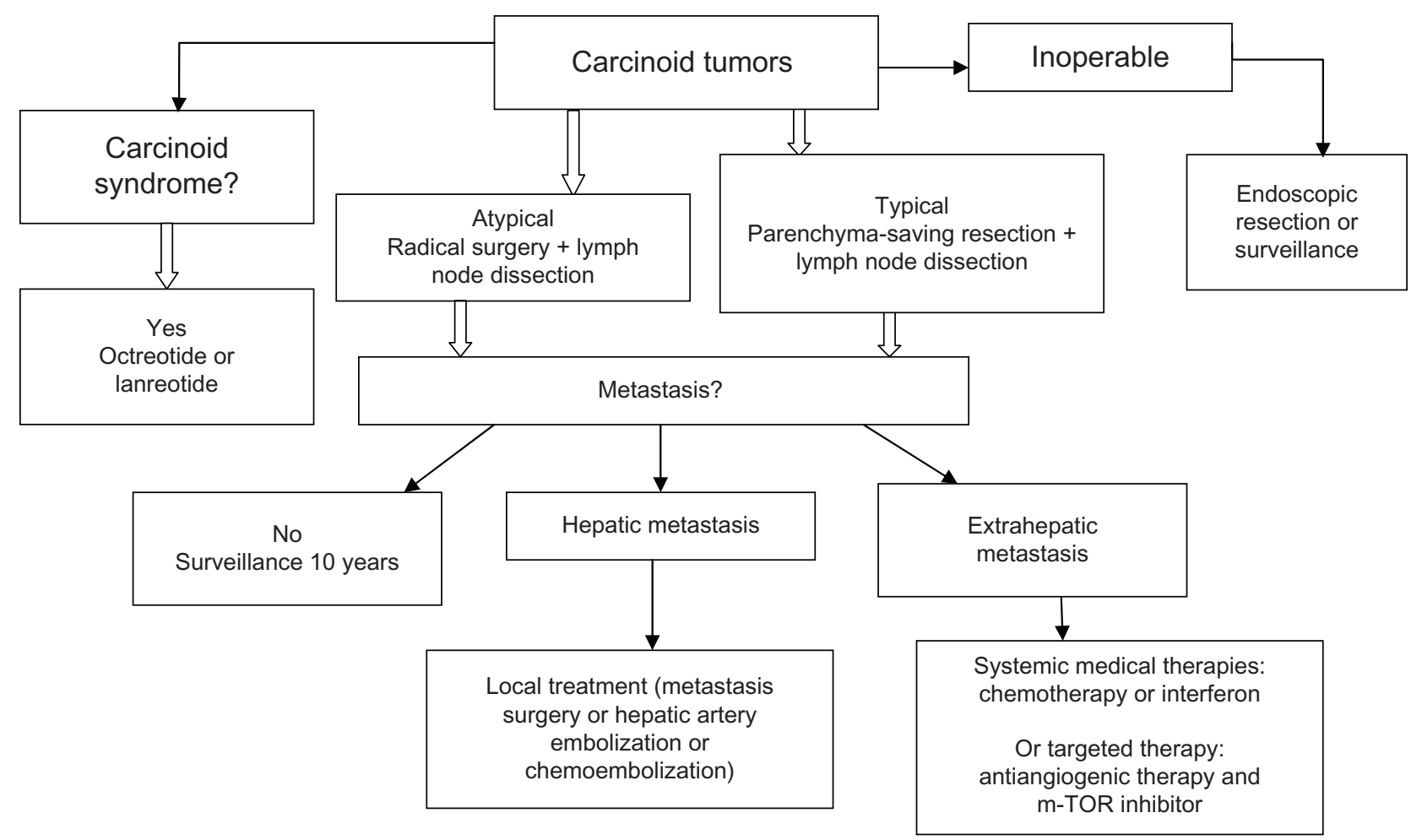

Figure I Treatment of carcinoid tumors. Proposed algorithm based on the type and stage of the tumor.

\section{Conclusion}

The treatment of choice for carcinoid tumors remains surgery and consists of a lobectomy supplemented by lymph node dissection. The benefit of chemotherapy and radiotherapy is unclear, but the question arises only for metastatic disease. Targeted therapy could be used in this indication, but there is a lack of studies to recommend these therapies. The treatment depends on the type of carcinoid tumor (typical or atypical), the presence of hepatic metastasis (local treatment) or extrahepatic metastasis, and the operability of the patient (Figure 1).

\section{Disclosure}

The authors report no conflicts of interest in this work.

\section{References}

1. Tsuta K, Raso MG, Kalhor N, Liu DD, Wistuba II, Moran CA. Histologic features of low- and intermediate-grade neuroendocrine carcinoma (typical and atypical carcinoid tumors) of the lung. Lung Cancer. 2011;71(1):34-41.

2. Morandi U, Casali C, Rossi G. Bronchial typical carcinoid tumors. Semin Thorac Cardiovasc Surg. 2006;18(3):191-198.

3. Travis. Pathology of lung cancer. Clin Chest Med; 2002:23-81.

4. Bouvier N, Zegerling V, Halley A. [Primitive metastasizing bronchial carcinoid with long survival]. Rev Mal Respir. 2007;24(1):63-68. French [with English abstract].

5. Iglesias M, Belda J, Baldó X, et al. [Bronchial carcinoid tumor: a retrospective analysis of 62 surgically treated cases]. Arch Bronconeumol. 2004;40(5):218-221. Spanish [abstract in English].
6. Ducrocq X, Thomas P, Massard G, et al. Operative risk and prognostic factors of typical bronchial carcinoid tumors. Ann Thorac Surg. 1998;65(5):1410-1414.

7. Bölükbas S, Schirren J. Parenchyma-sparing bronchial sleeve resections in trauma, benign and malign diseases. Thorac Cardiovasc Surg. 2010;58(1):32-37.

8. Aberg T, Blöndal T, Nõu E, Malmaeus J. The choice of operation for bronchial carcinoids. Ann Thorac Surg. 1981;32(1):19-22.

9. Machuca TN, Cardoso PF, Camargo SM. Surgical treatment of bronchial carcinoid tumors: a single-center experience. Lung Cancer. 2010;70(2):158-162.

10. García-Yuste M, Matilla JM, Cueto A, et al; Spanish Multi-centric Study of Neuroendocrine Tumours of the Lung for the Spanish Society of Pneumonology and Thoracic Surgery (EMETNE-SEPAR). Typical and atypical carcinoid tumours: analysis of the experience of the Spanish Multi-centric Study of Neuroendocrine Tumours of the Lung. Eur J Cardiothorac Surg. 2007;31(2):192-197.

11. Brokx HA, Risse EK, Paul MA, et al. Initial bronchoscopic treatment for patients with intraluminal bronchial carcinoids. JThorac Cardiovasc Surg. 2007;133(4):973-978.

12. Saji H, Tsuboi M, Matsubayashi J, et al. Clinical response of large cell neuroendocrine carcinoma of the lung to perioperative adjuvant chemotherapy. Anticancer Drugs. 2010;21(1): 89-93.

13. Sun W, Lipsitz S, Catalano P, Mailliard JA, Haller DG; Eastern Cooperative Oncology Group. Phase II/III study of doxorubicin with fluorouracil compared with streptozocin with fluorouracil or dacarbazine in the treatment of advanced carcinoid tumors: Eastern Cooperative Oncology Group Study E1281. J Clin Oncol. 2005;23(22): 4897-4904.

14. Pavel ME, Hainsworth JD, Baudin E, et al; RADIANT-2 Study Group. Everolimus plus octreotide long-acting repeatable for the treatment of advanced neuroendocrine tumours associated with carcinoid syndrome (RADIANT-2): a randomised, placebo-controlled, phase 3 study. Lancet. 2011;378(9808):2005-2012. 
15. Oberg K, Hellman P, Kwekkeboom D, Jelic S; ESMO Guidelines Working Group. Neuroendocrine bronchial and thymic tumours: ESMO Clinical Practice Guidelines for diagnosis, treatment and follow-up. Ann Oncol. 2010;21 Suppl 5:v220-v222.

16. Filosso PL, Ruffini E, Oliaro A, Papalia E, Donati G, Rena O. Long-term survival of atypical bronchial carcinoids with liver metastasis, treated with octreotide. Eur J Cardiothorac Surg. 2002;21(5):913-197.

17. Waldherr C, Pless M, Maecke HR, et al. Tumor response and clinical benefit in neuroendocrine tumors after $7.4 \mathrm{GBq}$ (90)Y-DOTATOC. J Nucl Med. 2002;43(5):610-616.

18. Bushnell DL Jr, O’Dorisio TM, O’Dorisio MS, et al. 90Y-endotreotide for metastatic carcinoid refractory to octreotide. J Clin Oncol. 2010;28(10):1652-1659.

19. Kulke MH, Lenz HJ, Meropol NJ, et al. Activity of sunitinib in patients with advanced neuroendocrine tumors. J Clin Oncol. 2008;26(20): 3403-3410.
20. Kölby L, Persson G, Franzén S, Ahrén B. Randomized clinical trial of the effect of interferon alpha on survival in patients with disseminated midgut carcinoid tumours. Br J Surg. 2003;90(6):687-693.

21. Yao JC, Phan A, Hoff PM, et al. Targeting vascular endothelial growth factor in advanced carcinoid tumor: a random assignment phase II study of depot octreotide with bevacizumab and pegylated interferon alpha-2b. J Clin Oncol. 2008;26(8):1316-1323.

22. Rickman OB, Vohra PK, Sanyal B, et al. Analysis of ErbB receptors in pulmonary carcinoid tumors. Clin Cancer Res. 2009;15(10): 3315-3324.

23. Diaco DS, Hajarizadeh H, Mueller CR, Fletcher WS, Pommier RF, Woltering EA. Treatment of metastatic carcinoid tumors using multimodality therapy of octreotide acetate, intra-arterial chemotherapy, and hepatic arterial chemoembolization. Am J Surg. 1995;169(5): 523-528.

\section{Publish your work in this journal}

OncoTargets and Therapy is an international, peer-reviewed, open access journal focusing on the pathological basis of all cancers, potential targets for therapy and treatment protocols employed to improve the management of cancer patients. The journal also focuses on the impact of management programs and new therapeutic agents and protocols on

\section{Dovepress}

patient perspectives such as quality of life, adherence and satisfaction. The manuscript management system is completely online and includes a very quick and fair peer-review system, which is all easy to use. Visit http://www.dovepress.com/testimonials.php to read real quotes from published authors.

Submit your manuscript here: http://www.dovepress.com/oncotargets-and-therapy-journal 